\title{
IAMVT: Mecanismo de Seleção de Rotas Multicaminhos para Aplicações de Vídeo Monitoramento
}

\author{
Fabiano Bhering ${ }^{1,2}$, Célio Albuquerque ${ }^{1}$, Diego Passos $^{1}$, Katia Obraczka $^{3}$ \\ ${ }^{1}$ Laboratório MídiaCom - Universidade Federal Fluminense (UFF) - Brasil \\ ${ }^{2}$ Centro Federal de Educação Tecnológica de Minas Gerais (CEFET-MG) - Brasil \\ ${ }^{3}$ University of California Santa Cruz (UCSC) - USA \\ fabianobhering@cefetmg.br, \{celio,dpassos\}@ic.uff.br, katia@soe.ucsc.edu
}

\begin{abstract}
Some of the main challenges for video transmission in wireless networks are related to bandwidth and high bitrate. Previous works indicate that dividing the video flows to transmit them over multiple paths can improve applications' QoS (Quality of Service). However, multipath selection is not a trivial task, especially when applications require simultaneous transmission from multiple sources, as it is necessary to find a set of paths with minimal inter-flow interference to support the applications' requirements. Thus, this paper presents Interference Aware Multipath Video Transmission (IAMVT), a multipath selection mechanism based on the Iterated Local Search (ILS) metaheuristic. This mechanism seeks to maximize the global network performance for transmitting multiple video flows from different sources. Simulation results show the potential of IAMVT to increase video quality, when compared to other mechanisms proposed in the literature.
\end{abstract}

Resumo. Alguns dos principais desafios para transmissão de vídeo em redes sem fio estão relacionados com a largura de banda e altas taxas de transmissão. Estudos indicam que dividir os fluxos do vídeo para transmiti-los por diferentes caminhos pode melhorar a qualidade do serviço (Quality of Service - QoS) das aplicações. Porém, a seleção de rotas multicaminhos não é uma tarefa trivial, principalmente quando as aplicações necessitam de múltiplas fontes de vídeo transmitindo simultaneamente, pois é necessário encontrar um conjunto de caminhos com o mínimo de interferência entre os fluxos para suportar os requisitos das aplicações. Assim, este trabalho apresenta o Interference Aware Multipath Video Transmission (IAMVT), um mecanismo de seleção de rotas multicaminhos baseado na metaheurística Iterated Local Search (ILS). O mecanismo busca maximizar o rendimento global da rede para transmissão de múltiplos fluxos de vídeos provindos de diferentes fontes. Os resultados das simulações demonstram o potencial da utilização do IAMVT para aumentar a qualidade do vídeo, quando comparado com outros mecanismos da literatura.

\section{Introdução}

A transmissão eficiente de vídeo em tempo real em redes sem fio é atualmente uma área ativa de pesquisa [Afzal et al. 2019]. O desempenho das aplicações depende de um protocolo de roteamento que identifique caminhos estáveis e confiáveis para garantir níveis 
adequados de qualidade do serviço (Quality of Service - QoS) [Hossain et al. 2019]. Com os novos codificadores escaláveis de vídeo, a transmissão concorrente de múltiplos fluxos por rotas multicaminhos tem sido fundamental para garantir a confiabilidade e a resiliência das aplicações [Hasan et al. 2017, Pan et al. 2016]. A técnica de roteamento multicaminhos é uma alternativa que seleciona diferentes caminhos para transmitir os diversos fluxos do vídeo, melhorando a robustez e o rendimento das transmissões. Para isso, o mecanismo de seleção de caminhos é primordial para otimizar o desempenho dos protocolos de roteamento, assim como para melhorar os recursos da rede por meio do balanceamento de carga entre os vários caminhos.

Existe uma grande variedade de cenários de aplicações que envolvem a transmissão de vídeo em tempo real em redes sem fio, tais como as emergentes aplicações Internet of Things (IoT) para cidades inteligentes, indústrias e reservas florestais. Os cenários de aplicações com múltiplas fontes de vídeo exigem um mecanismo de seleção de caminhos ainda mais complexo, pois precisam atender os requisitos das diversas fontes transmitindo simultaneamente. Uma das aplicações mais comuns para esses cenários é o vídeo monitoramento de ambientes internos ou externos para detectar eventos de interesse, no qual as múltiplas fontes de vídeo transmitem para uma determinada central [Dao et al. 2018]. Observe que o desempenho destas aplicações pode variar conforme a quantidade de fontes e a diversidade dos caminhos, pois os fluxos gerados por cada uma das fontes podem gerar interferência entre eles.

Com objetivo de prover um mecanismo para identificar um melhor conjunto de caminhos para suportar os cenários de vídeo monitoramento com transmissão de múltiplos fluxos provindos de diversas fontes, este trabalho propõe o Interference Aware Multipath Video Transmission (IAMVT), um mecanismo de seleção de rotas multicaminhos baseado na metaheurística Iterated Local Search (ILS) [Lourenço et al. 2010]. O mecanismo proposto adota uma otimização combinatória para selecionar caminhos com maior vazão, considerando as taxas de transmissão de cada fluxo e a interferência gerada entre eles. Resultados mostram que o IAMVT é capaz de selecionar caminhos que melhoram a qualidade de transmissão do vídeo, quando comparado com outros seis mecanismos propostos na literatura.

Este artigo está organizado da seguinte forma: inicialmente, a Seção 2 apresenta o problema; a Seção 3 apresenta os principais trabalhos relacionados; a Seção 4 apresenta o mecanismo proposto; a metodologia de avaliação e os resultados são apresentados nas Seções 5 e 6; por fim, a Seção 7 apresentada as conclusões e direcionamento para trabalhos futuros.

\section{Problema de Seleção de Rotas Multicaminhos para Transmissão de Vídeo}

O mecanismo de seleção de rotas multicaminhos tem como objetivo encontrar o melhor conjunto de caminhos para transmitir os fluxos que são gerados por cada fonte de vídeo. A quantidade de caminhos necessários pode variar conforme a quantidade de fontes que, por sua vez, podem gerar diferentes fluxos. Neste caso, é necessário selecionar $n P$ caminhos, em um conjunto $P$ de candidatos, para a transmissão dos fluxos. Assim, temos $n P=$ $\sum_{s \in S} f_{s}$ onde $S$ é o conjunto de fontes e $f_{s}$ a quantidade de fluxos gerados por cada fonte $s$. Um exemplo da transmissão de múltiplos fluxos de vídeo provindos de diferentes fontes para um destino é mostrado na Figura 1. Em cada nó fonte $s$ de $S$, o codificador de vídeo 
pode gerar $f_{s}$ fluxos. Esses fluxos devem ser transmitidos por um conjunto de caminhos por meio de uma rede sem fio de múltiplos saltos. No nó destino, o decodificador é responsável por sincronizar e mesclar os dados dos fluxos recebidos por cada fonte.

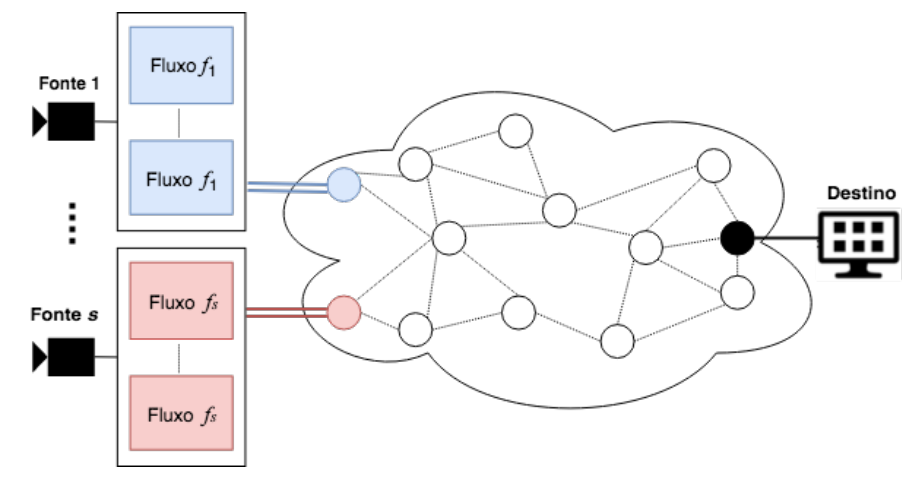

Figura 1. Transmissão de vídeo com múltiplas fontes e múltiplos caminhos.

A qualidade do vídeo entregue depende da vazão dos caminhos selecionados para cada fluxo. A vazão dos caminhos deve atender às taxas de transmissão dos fluxos, para reduzir as perdas e os atrasos. Para isso, o mecanismo de seleção de caminhos deve estar em conformidade com a estratégia de codificação de vídeo usada para gerar os fluxos. Existem basicamente duas estratégias: codificação por múltiplos descritores (Multiple Description Coding - MDC) e codificação em camadas (Layered Coding - LC). Com MDC, é possível particionar o vídeo em dois ou mais descritores, com taxas semelhantes, que podem ser decodificados de forma independente. Ou seja, uma qualidade aceitável do vídeo usando MDC pode ser obtida se os pacotes de pelo menos um dos fluxos não forem afetados [Begen et al. 2003]. LC tem sido implementado nos estabelecidos padrões de compressão de vídeo H.264/AVC e H.265/HEVC [Chakareski et al. 2003, Huo et al. 2015]. LC fornece fluxos de vídeos que são gerados em diferentes taxas de transmissão, particionando uma sequência de vídeo em uma hierarquia de camadas, onde as camadas superiores dependem da camada base. Assim, caminhos com maior vazão devem ser selecionados para garantir a entrega dos pacotes da camada base.

\section{Trabalhos Relacionados}

Existem diversos trabalhos que tratam da transmissão de vídeo por rotas multicaminhos em redes sem fio na literatura [Afzal et al. 2019, Hasan et al. 2017]. Nós selecionamos seis trabalhos para servir como referência para a proposta do IAMVT. Estes trabalhos foram escolhidos por representarem as diferentes abordagens e métricas adotadas nos mecanismos de seleção dos múltiplos caminhos.

\subsection{Mecanismos cientes de QoS e Energia}

O ERVT (Error Resilient Video Transmission) [Ghahremani and Ghanbari 2017] foi proposto para fluxos de vídeo gerados pela combinação das técnicas LC e MDC. O fluxo da camada base é duplicado e transmitido por diferentes caminhos para garantir a entrega dos quadros mais importantes do vídeo. Na seleção de rotas multicaminhos, uma versão estendida do protocolo de roteamento AOMDV [Marina and Das 2001] é implementada para identificar caminhos disjuntos. Para isso, o ERVT utiliza blocos de controle para 
obter um conjunto dos caminhos disjuntos ativos. Dois caminhos com melhor QoS deste conjunto são selecionados para a transmissão dos fluxos, enquanto os outros são considerados caminhos de backup. Essa abordagem pressupõe a existência de caminhos com nós disjuntos para cada fluxo. No entanto, essa suposição nem sempre é viável, principalmente para cenários de aplicações com várias fontes de vídeo.

O CLMR (Cross-Layer Multipath Routing) [Abazeed et al. 2018] foi proposto para transmitir os fluxos de vídeo gerados pela técnica LC por diferentes caminhos, de acordo com a prioridade das camadas. A descoberta dos caminhos é iniciada com pacotes de controle enviados da fonte para o destino. À medida que esses pacotes inundam a rede, os nós armazenam o custo de cada vizinho em sua tabela de roteamento. O nó de destino informa à fonte o custo total dos diversos caminhos. A função de custo adota uma média ponderada das métricas de atraso, vazão e energia, considerando a importância de cada uma. Assim, a fonte seleciona o conjunto de caminhos com menor custo. O mecanismo tem como objetivo garantir QoS e minimizar o consumo de energia. No entanto, os pesos da função são pré-definidos e configurados estaticamente, o que torna essa função não adequada para aplicações em tempo real em cenários dinâmicos.

\subsection{Mecanismos baseados em Posições Geográficas}

O RTVP (Real-Time Video Streaming Routing Protocol) [Ahmed 2017] também foi proposto para fluxos gerados pela técnica LC, priorizando os caminhos para os fluxos da camada base. O mecanismo de seleção de caminhos é implementado com base em uma estrutura geográfica em forma de coroas, na qual a rede é completamente dividida em determinados raios centrados no nó destino. Para cada nó, é atribuído um identificador do nível da coroa, conforme sua posição entre esses raios. Assim, os múltiplos caminhos são selecionados seguindo uma hierarquia de níveis entre a fonte e o destino, evitando os nós que compartilham os mesmos caminhos. Esta abordagem beneficia a mobilidade, mas dependendo da estrutura da coroa, pode selecionar caminhos com maior quantidade de saltos.

O Q-MMTP (QoS-oriented Multipath Multimedia Transmission Planning) [Hossain et al. 2019] propõe um algoritmo de geração de caminho usando o modelo matemático Spline. O algoritmo busca identificar uma determinada quantidade de caminhos uniformemente distribuídos no espaço, considerando os requisitos de atraso e a potência de transmissão dos nós. O Q-MMTP estima um conjunto de posições ideais para os nós, gerando uma série de interpolações entre a fonte e o destino. Assim, os caminhos são selecionando de acordo com a distância entre os nós e as posições ideais. Embora a estratégia Q-MMTP dependa da posição geográfica dos nós, ela considera o equilíbrio de energia-atraso apenas para uma das fontes. A proposta não especifica uma codificação de vídeo para os fluxos, mas inclui um escalonamento de caminho para distribuir os fluxos e balancear a carga da rede.

\subsection{Mecanismos baseados em Heurísticas}

O QSOpt (QoE-based Optimal Routing) [Pham et al. 2018] formula o problema de seleção de múltiplos caminho usando programação linear inteira mista. O mecanismo implementa uma heurística para encontrar uma solução baseada na qualidade de experiência do usuário (Quality of Experience - QoE). Para avaliar o QoE, é proposta uma função discreta para determinar um mapeamento entre as métricas de perdas de pacotes e QoE. 
Além disso, o QSOpt implementa um modelo de múltiplos fluxos de commodities com o objetivo de maximizar o QoE dos fluxos, com as restrições de atraso e capacidade dos enlaces. O mecanismo demonstra uma solução viável para melhorar a utilização de recursos, bem como o QoE. No entanto, adota um algoritmo centralizado que não é escalável e requer recursos computacionais significativos. O mecanismo também assume taxas de transmissão idênticas para todos os fluxos, não considerando as características da codificação do vídeo.

Recentemente, propusemos um mecanismo de seleção de rotas multicaminhos baseado na metaheurística ILS para transmissão de fluxos MDC, denominado ILSMDC [Bhering et al. 2019]. Esse mecanismo busca por uma combinação de caminhos que minimize a interferência entre os fluxos, com objetivo de maximizar a vazão agregada da rede. O mecanismo foi implementado usando o AFTER (Algorithmic Framework for Throughput EstimatoRs) [Passos and Albuquerque 2018], um estimador de vazão em tempo real para redes IEEE 802.11, considerando a visão global da rede. O AFTER funciona como um simulador determinístico de eventos discretos para prever a taxa de transferência de cada fluxo para um determinado conjunto de fluxos de dados e caminhos correspondentes. Assim, a heurística realiza buscas iterativas por caminhos com a maior vazão estimada. Porém, as taxas de transmissão não são especificadas no ILS-MDC, então o AFTER assume que cada fluxo transmitirá na taxa mais alta suportada pela rede. Como cada caminho potencialmente tem uma vazão máxima diferente dos demais, as taxas assumidas para os fluxos MDC serão provavelmente diferentes.

Assim como o ILS-MDC, o mecanismo proposto no presente artigo é baseado na metaheurística ILS, considerando a transmissão de múltiplos fluxos de vídeo provindas de diversas fontes. Além de um refinamento dos procedimentos de busca no algoritmo ILS, uma das principais diferenças do IAMVT é a proposta de um novo módulo para a função objetivo que considera o modelo de tráfego do vídeo, considerando as taxas específicas de cada fluxo. Com isso, as taxas de transmissão dos fluxos são usadas para guiar o ILS na travessia do espaço de busca, ou seja, consideram-se as possíveis combinações que atendem às demandas específicas de cada fluxo do vídeo. Assim, o IAMVT é flexível, suportando diferentes estratégias de transmissão usadas com as técnicas MDC e/ou LC.

\section{Mecanismo IAMVT}

O IAMVT é um mecanismo de seleção de caminhos para transmissão simultânea de múltiplos fluxos de vídeos gerados por diversas fontes. Este mecanismo é baseado na metaheurística ILS com o objetivo de identificar um conjunto de caminhos com maior vazão, ciente da interferência entre os fluxos.

\subsection{Metaheurística Iterated Local Search (ILS)}

Metaheurísticas ainda são pouco exploradas para roteamento multicaminhos, mesmo se apresentando como alternativa para melhorar o desempenho de soluções com natureza similar [Neves et al. 2015]. O ILS, em especial, tem se apresentado como uma das melhores alternativas heurísticas para resolver diferentes problemas de otimização combinatória da classe NP-completo [Lourenço et al. 2010]. Sua eficiência e simplicidade têm demonstrado o potencial para resolver problemas de roteamento de rede [Penna et al. 2017, Lemamou et al. 2016], assim como para selecionar rotas multicaminhos para transmissão de múltiplas fontes de vídeo [Bhering et al. 2019]. 
O Algoritmo 1 mostra os procedimentos básicos do ILS usados na proposta do IAMVT, consistindo em: (i) SoluçãoInicial $p_{0}$; (ii) BuscaLocal para identificar uma melhor solução $p$; (iii) Perturbação para gerar soluções intermediárias $p^{\prime}$ e $p^{\prime \prime}$ e (iv) CritérioAceitação que determina se uma nova solução é aceita. Esses procedimentos iteram até que o Critério de Parada seja atendido, geralmente consistindo em um limite para a quantidade de iterações sem melhorias significativas e/ou um critério de tempo para interromper a busca, de acordo com os requisitos da aplicação. O CritérioAceitação tem forte influência na eficácia da busca no conjunto de soluções candidatas $P$. Assim, diferentes critérios podem ser usados para controlar o equilíbrio entre a intensificação (inspecionar minuciosamente a busca local) e a diversificação (explorar minuciosamente o espaço de solução) dessa busca.

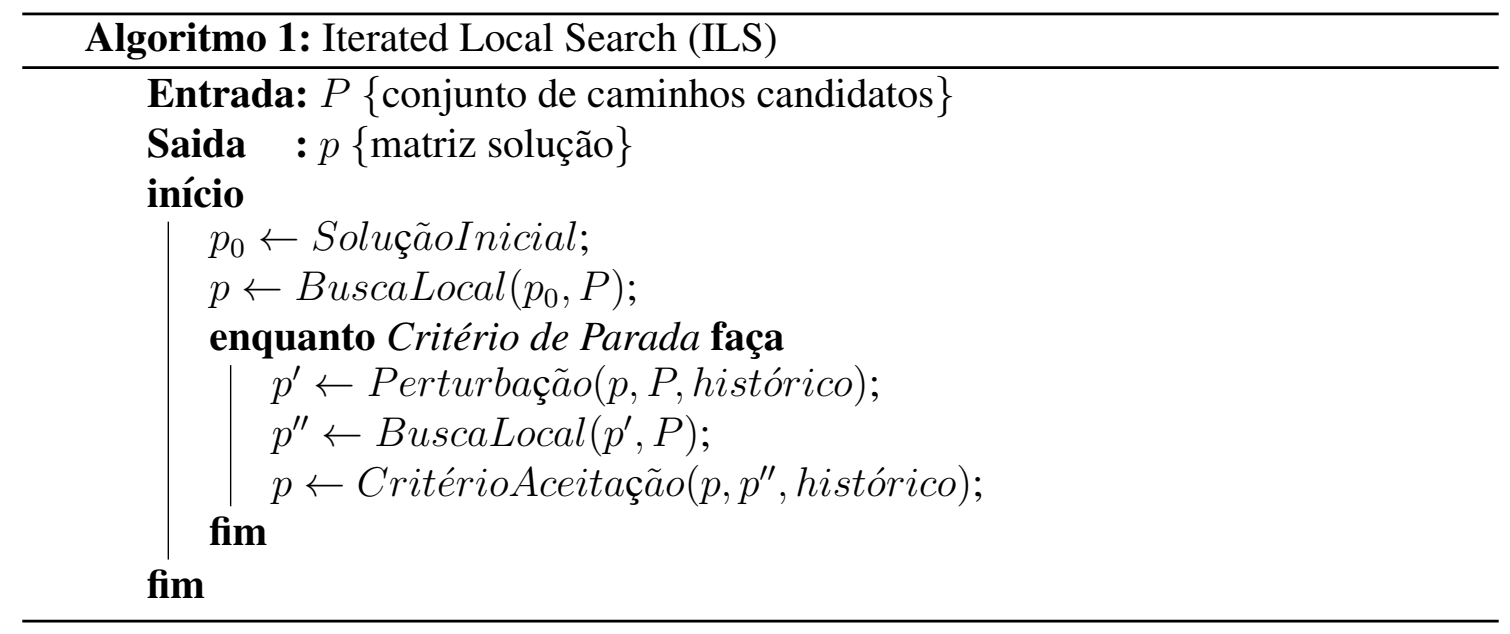

\subsection{Projeto e Implementação}

O IAMVT é responsável por selecionar um conjunto de caminhos com o menor custo para a transmissão dos fluxos, de acordo com as taxas geradas pela codificação do vídeo. $\mathrm{O}$ mecanismo recebe como entradas um conjunto de caminhos candidatos $P$, que é gerado a partir da topologia de rede representada pelo grafo $G(N, L, F, R)$ onde $N$ é um conjunto de nós $\{0,1 \ldots n\}, L$ é um conjunto de links $\{0,1 \ldots l\}, F$ é uma lista de fluxos associados às suas fontes e $R$ é uma lista das taxas de transmissão associadas a cada fluxo. O grafo $G$ pode ser obtido por um algoritmo de monitoramento do estado de enlace da rede, semelhante aos implementados em protocolos de roteamento proativo.

A Figura 2 mostra um exemplo de topologia de rede com duas fontes de vídeo representadas pelos nós 1 e 2 e um destino representado pelo nó 0 . Neste caso, se a codificação gerar dois fluxos para cada fonte, será necessário selecionar quatro caminhos para que todos os fluxos sejam transmitidos. Os procedimentos do Algoritmo 1 implementados para o IAMVT são detalhados a seguir.

\subsubsection{Solução Inicial}

O procedimento SoluçãoInicial retorna uma matriz $p$ de dimensões $n S \times n P$, onde $n S$ é o número de fontes e $n P$ é o número de caminhos requeridos para os fluxos de todas fontes. A solução inicial $p_{0}$ é composta por caminhos mínimos de cada fonte para o destino 


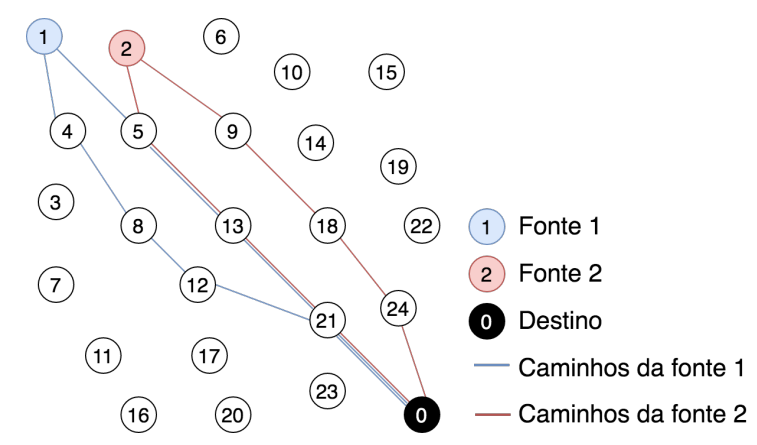

Figura 2. Exemplo de topologia com uma solução inicial de caminhos para a transmissão de duas fontes de vídeo com dois fluxos cada.

com base no algoritmo Dijkstra. A Figura 3 ilustra a possível matriz solução $p_{0}$ exibida na topologia da Figura 2. Cada célula da matriz representa um caminho para um determinado fluxo. Neste exemplo, os primeiros caminhos das fontes 1 e 2 compartilham os mesmos nós, o que pode causar interferência entre fluxos e, consequentemente, diminuir a vazão dos caminhos. Assim, as próximas etapas do mecanismo buscam iterativamente por novas soluções com o objetivo de maximizar essa métrica.

$$
p_{0}=\left[\begin{array}{cc}
{[1,5,13,21,0]} & {[1,4,8,12,21,0]} \\
{[2,5,13,21,0]} & {[2,9,18,24,0]}
\end{array}\right]
$$

Figura 3. Matriz para a Solução Inicial.

\subsubsection{Busca Local}

Neste trabalho, foi definida uma busca local simples, com complexidade $O(n \cdot \log n)$, para ser viável em dispositivos com média capacidade de processamento. O procedimento BuscaLocal procura em cada iteração os vizinhos mais próximos da solução atual $p$ para obter melhores soluções. Para isso, cada caminho na solução $p$ é substituído por um novo caminho do conjunto $P$. O Algoritmo 2 mostra o procedimento usando a função novoCaminho, onde $s$ é a fonte referente ao caminho a ser substituído. As soluções candidatas são avaliadas pelo procedimento vazãoEstimada, que estima a vazão dos caminhos da solução candidata, por meio de uma função objetivo.

\subsubsection{Função Objetivo}

Para implementar a função objetivo vazãoEstimada, estamos propondo um estimator de vazão agregada da rede baseado no AFTER [Passos and Albuquerque 2018]. Diferente do mecanismo ILS-MDC [Bhering et al. 2019], nós implementamos um novo módulo para o AFTER lidar com a taxa de transmissão específica de cada fluxo. Assim, além de utilizar as taxas de transmissão específicas da codificação do vídeo como referência, é possível estimar a vazão individual de cada um dos caminhos.

A função objetivo recebe como argumento as taxas de todos os fluxos e o conjunto de caminhos a ser avaliado, e simula a dinâmica da rede: (1) gerando pacotes para 


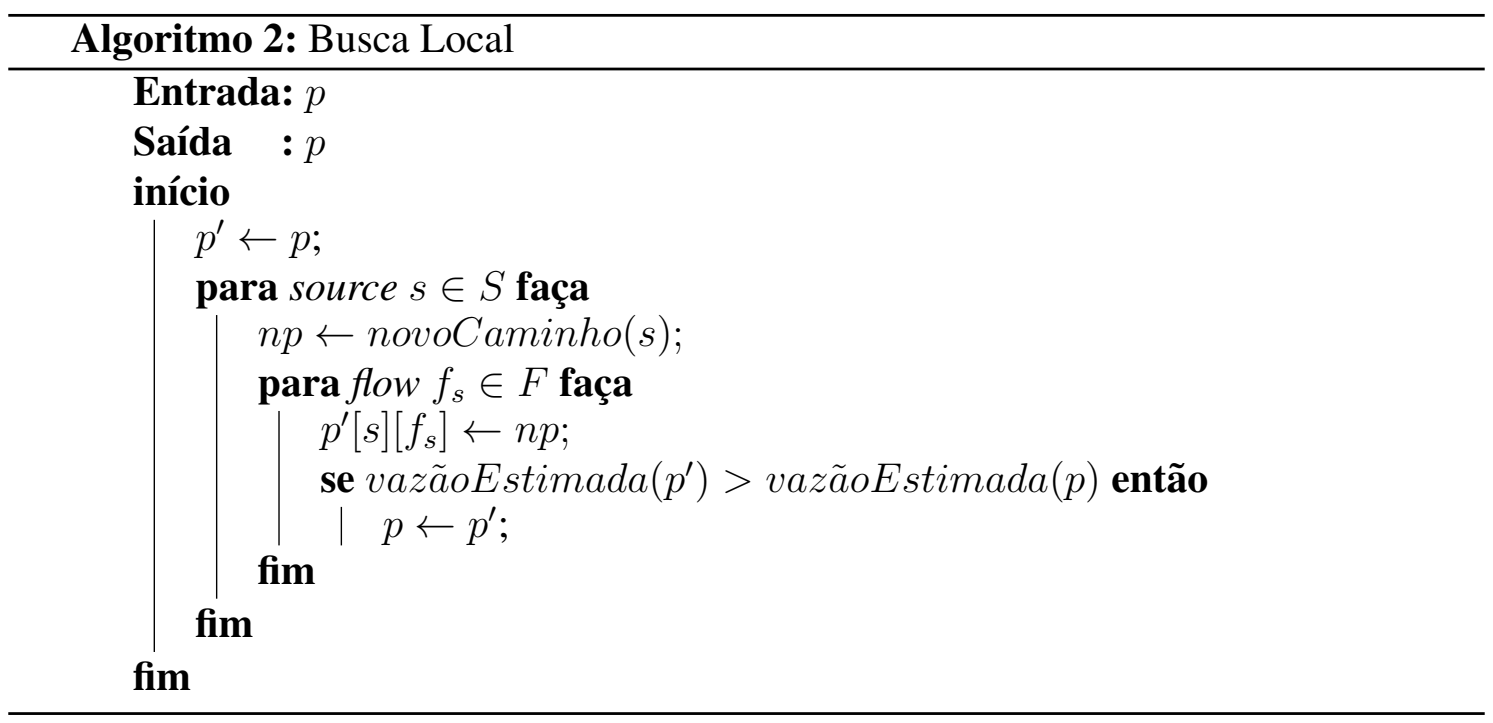

cada fluxo em seus respectivos nós fontes, (2) disparando uma série de outros eventos de simulação relevantes, como o acesso ao meio para a transmissão sem fio, gerenciamento de fila (pacotes sendo adicionados, removidos e descartados de buffers) e, (3) eventualmente, entrega de pacotes a seus nós destino. Como tal, a interferência entre fluxos ocorre como resultado de estouro de buffers, perdas de transmissão na camada de enlace e conflitos de acesso ao meio.

Assim, sempre que invocada, a função objetivo estima em tempo real a vazão dos caminhos, considerando a interferência entre os fluxos. Além disso, a função verifica se os caminhos irão ou não lidar satisfatoriamente com os fluxos que estão sendo gerados, retornando um custo para a transmissão destes fluxos na rede. Este custo representa a diferença percentual média entre a vazão estimada e a taxa de transmissão de cada fluxo. Ou seja, um custo 0 significa que todos os caminhos possuem a vazão suficiente para suportar plenamente os fluxos.

\subsubsection{Perturbação}

O procedimento Perturbação visa escapar de soluções ótimas locais de baixa qualidade, evitando com isso uma convergência prematura. Este procedimento foi implementado para gerar novos candidatos em cada iteração. Com isso, o mecanismo utiliza a solução atual, que perturba com as melhores soluções encontradas até o momento, armazenadas em um histórico, para obter um novo candidato. Para este fim, é executada uma rotina para gerar uma nova solução, que substitui aleatoriamente um dos caminhos de cada fonte.

\subsubsection{Critério de Aceitação}

O procedimento CritérioAceitação define um critério para aceitar ou não as soluções retornadas pela busca local $p^{\prime \prime}$, após a perturbação. O mecanismo tem como objetivo aceitar somente a melhor solução, comparada às soluções anteriores. Caso uma solução seja aceita, a solução atual é armazenada em um histórico que servirá como base para a 
perturbação na próxima iteração. A função objetivo também é utilizada neste procedimento para avaliar a qualidade das soluções com base na vazão dos caminhos.

O tempo de convergência do IAMVT depende da topologia da rede e dos cenários da aplicação, pois o espaço de busca aumenta conforme a quantidade de caminhos disponíveis. Para cenários mais complexos que requerem um maior tempo de convergência, os protocolos de roteamento que venham a adotar o IAMVT poderão usar uma estratégia para iniciar a transmissão dos fluxos do vídeo sem a necessidade de esperar o tempo de convergência. Neste caso, a transmissão poderia ser iniciada com a solução aceita na primeira iteração, possivelmente ainda distante da solução ótima, enquanto o mecanismo continua rodando em segundo plano, buscando por melhores soluções. Assim, à medida que as melhores soluções são encontradas, a transmissão dos fluxos torna-se mais eficiente, melhorando a qualidade do vídeo no decorrer do tempo até estabilizar com o término da execução do algoritmo.

\section{Metodologia de Avaliação}

Com o objetivo de demonstrar a eficácia do IAMVT, em relação às demais propostas identificadas na literatura, nós implementamos os seis mecanismos apresentados na Seção 3: RTVP, CLMR, ERVT, Q-MMTP, QSOpt e ILS-MDC. Além disso, nós avaliamos o IAMVT usando diferentes estratégias para gerar os fluxos: MDC e LC.

Usando o simulador ns-3, cada um dos mecanismos foi avaliado na simulação da transmissão de vídeo usando uma única topologia de rede sem fio gerada aleatoriamente. Essa topologia, exemplificada na Figura 3, possui 25 nós distribuídos em uma área de $400 \times 400 \mathrm{~m}$. Reproduzimos um cenário de aplicação típico dos sistemas de monitoramento [Natarajan et al. 2015], utilizando duas fontes de vídeo vizinhas, onde cada fonte transmite dois fluxos de vídeo para o mesmo destino. As fontes e o destino foram atribuídos aos nós da extremidade da topologia, aumentando a diversidade de caminhos para facilitar a análise dos mecanismos. Na configuração, foi adotado o modelo de propagação Cost 231 (Okumura-Hata) e o modelo de rede IEEE 802.11g em nível MAC e PHY com taxa fixa de $18 \mathrm{Mb} / \mathrm{s}$ na camada de enlace, buscando aproximar as aplicações implantadas em ambientes urbanos reais e de forma que os links resultantes da topologia suportem cenários das aplicações multimídia.

Para simular a transmissão do vídeo, foi utilizado o framework Evalvid [Klaue et al. 2003] que fornece ferramentas para gerar tráfegos de vídeo. Nos experimentos, nós utilizamos a sequência de vídeo Bus transmitindo 9000 quadros, por representar um padrão de sequência de um sistema de monitoramento em cidades inteligentes com alta complexidade de movimento das cenas [Seeling and Reisslein 2011]. O tráfego foi gerado conforme as estratégias de codificação especificadas por cada um dos trabalhos, considerando as técnicas MDC e LC no formato H.264/MPEG-4, com taxas de transmissão de $1024 \mathrm{~kb} / \mathrm{s}$ e pacotes de 1024 bytes.

Evalvid também fornece ferramentas para decodificar e mensurar a qualidade da entrega dos vídeos pelas simulações no ns-3. O índice de similaridade estrutural SSIM (Structural Similarity Index Measure) e a relação sinal-ruído de pico PSNR (Peak Signalto-Noise Ratio) são as principais métricas objetivas para este propósito. O SSIM mede a distorção estrutural do vídeo, que pode ser correlacionada à percepção subjetiva de QoE. Para isso, combina luminância, contraste e semelhança estrutural dos quadros para 
comparar o quadro original com o entregue (possivelmente distorcido). Os valores SSIM variam entre 0 e 1 , onde 1 significa a qualidade máxima. O PSNR, representado em $d B$, é calculado pelo erro quadro a quadro entre o video original e o entregue. $\mathrm{O}$ valor do PSNR tende a aumentar à medida que a qualidade do vídeo melhora. Além das métricas de qualidade, também foram avaliados o atraso, a vazão e as perdas de pacotes de cada fonte de transmissão.

\section{Resultados Experimentais}

Esta seção apresenta os resultados dos experimentos realizados. Primeiramente, nós analisamos os caminhos selecionados por cada um dos mecanismos avaliados. Em seguida, comparamos as métricas de qualidade do vídeo e da rede obtidas com as simulações.

\subsection{Análise dos Caminhos Selecionados}

A Figura 4 ilustra o conjunto de caminhos selecionados por cada um dos mecanismos, considerando os dois fluxos de vídeo de cada fonte. Podemos observar que os mecanismos cientes de QoS e energia, ERVT e CLMR, encontraram dois caminhos disjuntos para cada uma das fontes, mas, como as fontes são vizinhas, há muitos links e nós compartilhados entre fluxos de fontes diferentes, o que pode levar a interferência.

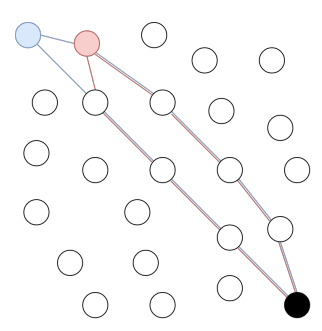

(a) ERVT

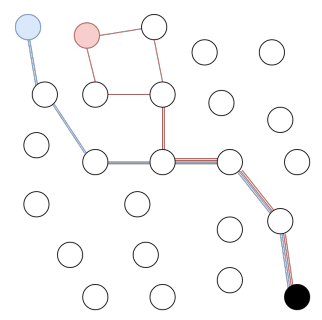

(e) QSOpt

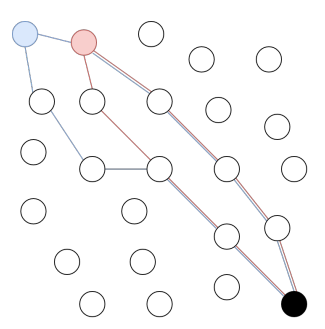

(b) CLMR

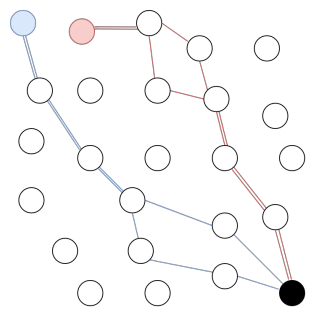

(f) ILS-MDC

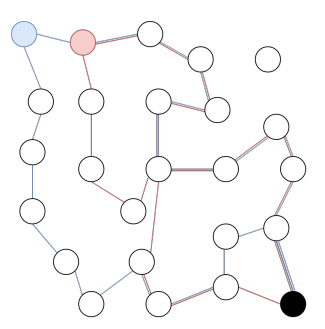

(c) RTVP

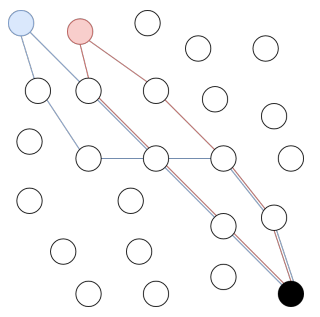

(g) IAMVT-MDC

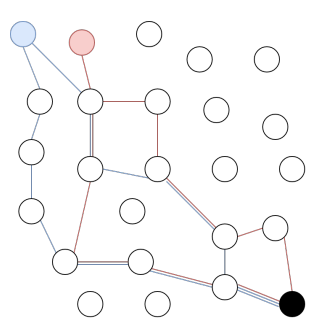

(d) Q-MMTP

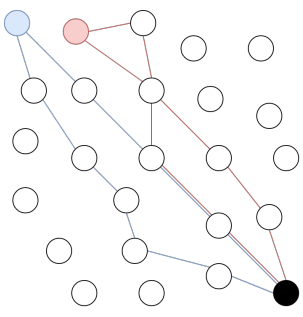

(h) IAMVT-LC

Figura 4. Comparação entre os Caminhos Selecionados pelos mecanismos dos trabalhos relacionados e pela proposta IAMVT.

Os mecanismos baseados em posições geográficas conseguiram reduzir a interferência entre os fluxos. A estrutura de coroas e a função de custo dinâmica do RTVP diversificaram os caminhos, priorizando os caminhos disjuntos e seguindo os níveis das coroas em direção ao destino. No entanto, essa abordagem gerou caminhos com muitos saltos, podendo aumentar o atraso fim-a-fim. O Q-MMTP conseguiu uma melhor distribuição geográfica entre os caminhos ao se basear nos caminhos virtuais gerados pelo modelo Spline. No entanto, como acontece com a maioria dos mecanismos avaliados, o Q-MMTP também não considera múltiplas fontes, resultando em possíveis interferências entre os fluxos. 
O QSOpt, ILS-MDC e o IAMVT são os únicos mecanismos que implementam heurísticas para levar em consideração a interferência entre fluxos de múltiplas fontes. O QSOpt adota a programação linear inteira mista sob as restrições de tempo e capacidade de enlace para selecionar os múltiplos caminhos. Porém, quando a soma das taxas de todos os fluxos é menor que a capacidade dos links, o QSOpt tende a selecionar os caminhos mínimos para todas as fontes, podendo sobrecarregar os nós e gerar perdas de pacotes. É possível visualizar claramente que os últimos três saltos dos quatro caminhos selecionados por esta proposta são idênticos. Por outro lado, o ILS-MDC considera a interferência entre fluxos buscando maximizar a vazão agregada da rede, resultando em uma melhor distribuição dos fluxos. Porém, o mecanismo considera uma taxa máxima de transmissão para os fluxos, podendo encontrar soluções com vazão agregada alta, mas que não atendam plenamente às taxas requeridas para todos os fluxos.

A heurística do IAMVT selecionou os caminhos conforme a estimativa da vazão dos fluxos adotada pela função objetivo, usando as taxas geradas pela codificação do vídeo como referência. Considerando taxas iguais para todos fluxos, o IAMVT-MDC apresentou uma diversificação dos caminhos com menor quantidade de saltos. Como esperado, ao especificar as taxas geradas por LC, o IAMVT-LC encontrou uma melhor distribuição dos caminhos, diminuindo a quantidade nós compartilhados sem aumentar significativamente a quantidade de saltos. Ou seja, além de minimizar a interferência, o mecanismo busca por caminhos que satisfazem as taxas requeridas pelos fluxos.

\subsection{Avaliação de Desempenho}

A Figura 5 apresenta a média do SSIM para os quadros entregues e do atraso fim-a-fim com intervalo de confiança de $95 \%$, a vazão obtida pelo destino e a taxa percentual de perdas de pacotes para cada fonte dos mecanismos avaliados. Avaliando o desempenho do SSIM, podemos observar que, devido ao posicionamento das fontes e ao congestionamento gerado pelos fluxos concorrentes, a fonte 1 foi prejudicada em todos os mecanismos. O IAMVT-MDC e IAMVT-LC obtiveram os maiores níveis de qualidade para as duas fontes entre todos os mecanismos, ambos atingindo $98 \%$ similaridade para a fonte 2. No entanto, o IAMVT-MDC teve um desempenho inferior para a fonte 1 devido à sobrecarga em nós compartilhados gerada por fluxos com as mesmas taxas de transmissão. Os mecanismos ERVT e ILS-MDC também obtiveram o SSIM competitivo para a fonte 2 , mas com desempenho muito inferior para fonte 1. Isso ocorre porque o ERVT selecionou caminhos com bons links, mas com alta interferência entre os fluxos. Já o ILS-MDC identificou caminhos com menor interferência, mas a solução com maior vazão agregada não foi suficiente para atender os fluxos da fonte 1 .

As duas estratégias de codificação do IAMVT tiveram o atraso médio menor que $180 \mathrm{~ms}$ para a fonte 2, superando todos os demais mecanismos. No entanto, o atraso da fonte 1 foi maior e mais equilibrado entre a maioria dos mecanismos. Somente o Q-MMTP obteve um atraso menor que $250 \mathrm{~ms}$. O mecanismo adota a distribuição dos caminhos com base no atraso, mas não conseguiu manter o bom desempenho na fonte 2 .

A vazão e as taxas de perdas obtidas pelos caminhos de cada mecanismo refletem os resultados do SSIM. As duas versões do IAMVT foram capazes de selecionar caminhos que forneceram a maior vazão para os vídeos, atingindo praticamente a mesma taxa usada para a codificação na fonte 2. O CLMR foi único mecanismo que obteve resultados nesses 

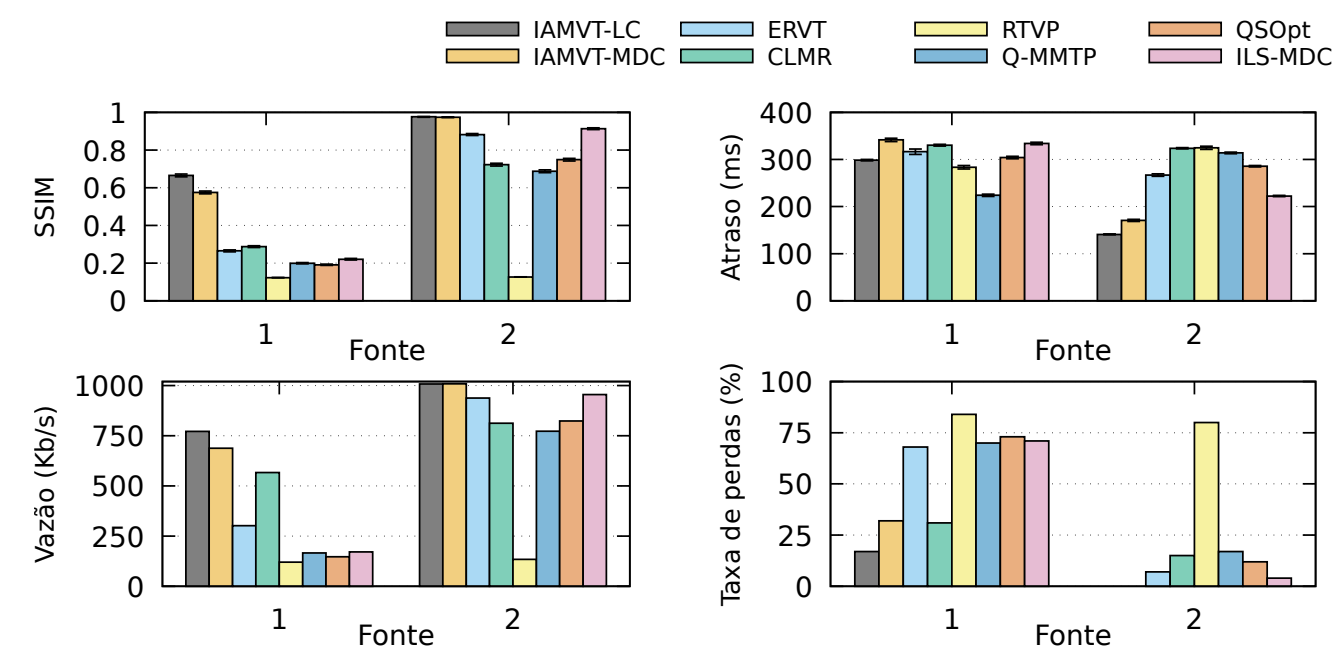

Figura 5. Comparação entre SSIM, atraso, vazão e taxa de perdas para cada fonte.

dois quesitos que não refletiram integralmente a qualidade do vídeo para a fonte 1, devido principalmente a quadros que foram perdidos no início da transmissão.

Além das métricas de rede avaliadas, o SSIM também depende dos recursos da codificação. Alguns decodificadores podem processar um quadro mesmo se algumas partes estiverem faltando, enquanto outros não. A Figura 6 demonstra o PSNR da sequência de quadros transmitidos por cada uma das fontes. O IAMVT-LC e IAMVT-MDC apresentaram melhor qualidade do vídeo durante toda transmissão, obtendo maiores níveis PSNR para as duas fontes. Na fonte 2, os dois mecanismos atingiram níveis máximos no início da transmissão e ficaram sobrepostos em $38 d B$ no restante dos quadros. Embora todos mecanismos apresentem PSNR instável no início da transmissão por motivo das rajadas geradas pelos primeiros quadros do vídeo, podemos observar que esses níveis estabilizam ao longo da transmissão.
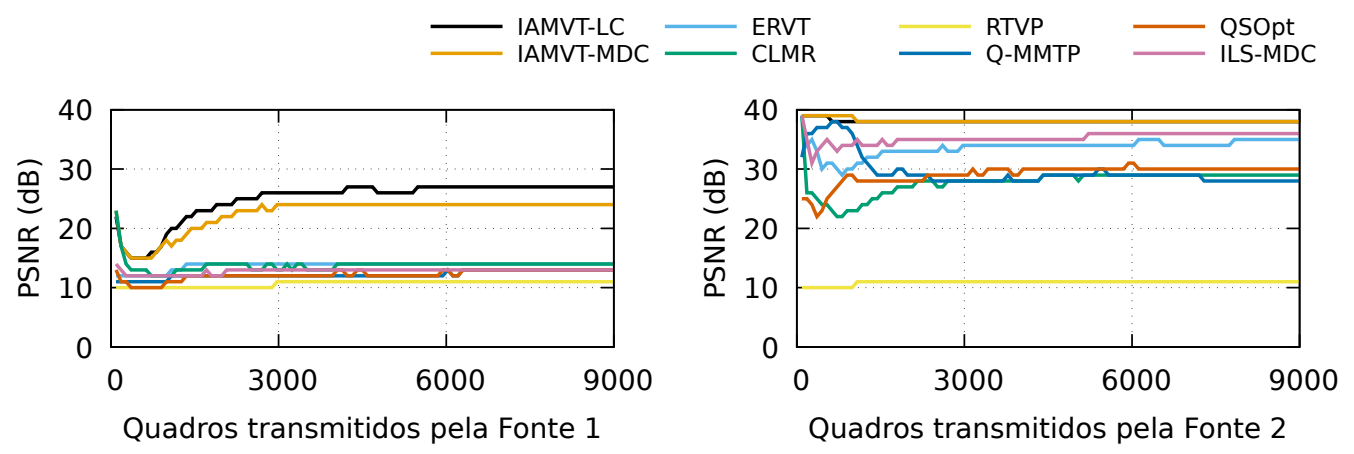

Figura 6. Comparação do PSNR durante a transmissão dos quadros de cada fonte.

Pelos resultados, podemos constatar que a qualidade da entrega do vídeo está relacionada principalmente à vazão dos caminhos. Portanto, a seleção de caminhos baseada na estimativa da vazão adotada pelo IAMVT, considerando as taxas específicas de cada fluxo do vídeo para as estratégias de MDC e LC, apresentou o melhor desempenho e convergiu rapidamente para a melhor qualidade do vídeo em relação aos demais mecanismos avaliados. 


\section{Conclusão}

A transmissão de vídeo por rotas multicaminhos tem sido fundamental para melhorar o QoS das aplicações. No entanto, o mecanismo de seleção de caminhos requer grande esforço computacional para considerar as interferências entre os fluxos de diversas fontes, devido à diversidade de soluções. Nesse sentido, este trabalho apresentou o IAMVT, um mecanismo baseado na metaheurística ILS, incluindo a proposta de uma função objetivo para estimar o custo dos caminhos levando em consideração as taxas de transmissão dos fluxos. Foram implementados outros seis mecanismos com objetivo de avaliar o desempenho da proposta. Os resultados obtidos por cada um dos mecanismos demonstram o potencial do IAMVT para melhorar a qualidade da transmissão dos vídeos, usando as diferentes técnicas de codificação.

Para trabalhos futuros, pretendemos investigar versões estendidas do IAMVT, introduzindo novas estratégias de busca local e perturbação, com diferentes estruturas de vizinhança. Outra proposta inclui incorporar o suporte para codificação de vídeo adaptativa, além de investigar a correlação entre as métricas de rede e o SSIM para otimizar a função objetivo. Por fim, o mecanismo deve ser avaliado em topologias de redes com diferentes densidades e para os diferentes cenários de aplicações.

\section{Referências}

Abazeed, M., Faisal, N., and Ali, A. (2018). Cross-layer multipath routing scheme for wireless multimedia sensor network. Wireless Networks, 6.

Afzal, S., Testoni, V., Rothenberg, C. E., Kolan, P., and Bouazizi, I. (2019). A Holistic Survey of Wireless Multipath Video Streaming. IEEE Communications Surveys and Tutorials, pages 1-42.

Ahmed, A. A. (2017). A real-time routing protocol with adaptive traffic shaping for multimedia streaming over next-generation of Wireless Multimedia Sensor Networks. Pervasive and Mobile Computing, 40:495-511.

Begen, A. C., Altunbasak, Y., and Ergun, O. (2003). Multi-path selection for multiple description encoded video streaming. In IEEE International Conference on Communications (ICC), volume 3, pages 1583-1589. IEEE.

Bhering, F., Albuquerque, C., Passos, D., and Ochi, L. S. (2019). Multipath selection mechanism for wireless video-surveillance systems. 9th Latin American Network Operations and Management Symposium, LANOMS.

Chakareski, J., Han, S., and Girod, B. (2003). Layered coding vs. multiple descriptions for video streaming over multiple paths. Proceedings of the ACM International Multimedia Conference and Exhibition, 285:422-431.

Dao, N. Q., Le-Dang, Q., Morawski, R., Dang, A. T., and Le-Ngoc, T. (2018). Management of video surveillance for smart cities. Handbook of Smart Cities: Software Services and Cyber Infrastructure, pages 285-310.

Ghahremani, S. and Ghanbari, M. (2017). Error resilient video transmission in ad hoc networks using layered and multiple description coding. Multimedia Tools and Applications, 76(6):9033-9049. 
Hasan, M. Z., Al-Rizzo, H., and Al-Turjman, F. (2017). A Survey on Multipath Routing Protocols for QoS Assurances in Real-Time Wireless Multimedia Sensor Networks. IEEE Communications Surveys and Tutorials, 19(3):1424-1456.

Hossain, M. S., You, X., Xiao, W., Lu, J., and Song, E. (2019). QoS-oriented multimedia transmission using multipath routing. Future Generation Computer Systems, 99:226234.

Huo, Y., Hellge, C., Wiegand, T., and Hanzo, L. (2015). A tutorial and review on interlayer fec coded layered video streaming. IEEE Communications Surveys and Tutorials, 17(2):1166-1207.

Klaue, J., Rathke, B., and Wolisz, A. (2003). Evalvid-a framework for video transmission and quality evaluation. In International conference on modelling techniques and tools for computer performance evaluation, pages 255-272. Springer.

Lemamou, E. A., Galinier, P., and Chamberland, S. (2016). A hybrid iterated local search algorithm for the global planning problem of survivable $4 \mathrm{~g}$ wireless networks. IEEE/ACM Transactions on Networking (TON), 24(1):137-148.

Lourenço, H. R., Martin, O. C., and Stützle, T. (2010). Iterated local search: Framework and applications. In Handbook of metaheuristics, pages 363-397. Springer.

Marina, M. K. and Das, S. R. (2001). On-demand multipath distance vector routing in ad hoc networks. In Proceedings Ninth International Conference on Network Protocols. ICNP 2001, pages 14-23. IEEE.

Natarajan, P., Atrey, P. K., and Kankanhalli, M. (2015). Multi-camera coordination and control in surveillance systems: A survey. ACM Transactions on Multimedia Computing, Communications, and Applications (TOMM), 11(4):57.

Neves, T., Ochi, L. S., and Albuquerque, C. (2015). A new hybrid heuristic for replica placement and request distribution in content distribution networks. Optimization Letters, 9(4):677-692.

Pan, C., Liu, B., Zhou, H., and Gui, L. (2016). Multi-path routing for video streaming in multi-radio multi-channel wireless mesh networks. 2016 IEEE International Conference on Communications, ICC 2016, 11:73-92.

Passos, D. and Albuquerque, C. (2018). AFTER: Algorithmic Framework for Throughput EstimatoRs for IEEE 802.11 networks. Simulation Modelling Practice and Theory, 84:143-160.

Penna, P. H. V., Subramanian, A., Ochi, L. S., Vidal, T., and Prins, C. (2017). A hybrid heuristic for a broad class of vehicle routing problems with heterogeneous fleet. Annals of Operations Research, pages 1-70.

Pham, T. A. Q., Singh, K. D., Rodríguez-Aguilar, J. A., Picard, G., Piamrat, K., Cerquides, J., and Viho, C. (2018). AD 3 -GLAM: A cooperative distributed QoE-based approach for SVC video streaming over wireless mesh networks. Ad Hoc Networks, $80: 1-15$.

Seeling, P. and Reisslein, M. (2011). Video transport evaluation with h. 264 video traces. IEEE Communications Surveys \& Tutorials, 14(4):1142-1165. 